\title{
Altimeter sampling characteristics using a single satellite
}

\author{
Michael E. Parke, ${ }^{1}$ George Born, Robert Leben, Craig McLaughlin, and \\ Craig Tierney \\ Colorado Center for Astrodynamics Research, University of Colorado at Boulder
}

\begin{abstract}
Altimetric satellites have characteristic sampling patterns in both space and time based on their repeat period and orbit inclination. Aliased phenomena measured by altimetric measurements can appear as propagating waves with both wavelength and direction of propagation different from the underlying phenomena. All signals that contribute to the altimetric measurement can be aliased and produce such patterns, not just tidal signals. For example, mesoscale energy will be aliased as will unmodeled atmospheric variations. Past discussions of aliasing have only considered spatially homogeneous signals. This paper extends this work to phenomena with finite wavelengths and considers both the north-south and east-west components of the resulting aliases.
\end{abstract}

\section{Introduction}

Aliasing of oceanographic phenomena has long been a concern of the altimetric and oceanographic communities. Temporal aliasing of tides was one of the driving considerations in the choice of the orbit for the TOPEX/POSEIDON (T/P) mission [Parke et al., 1987]. The $T / P$ orbit will also be used by Jason 1 . The primary goal of $T / P$, to measure basin scale ocean circulation, dictated a shorter repeat period than Geosat with a correspondingly coarser spatial resolution. Other satellite orbits commonly used in oceanographic research are the 17 day repeat orbit used by the Geosat Exact Repeat Mission and Geosat Follow-On Mission (Geosat) and the 35 day repeat orbit used by ERS-1/2 (ERS).

Most historical work has considered only tidal aliasing. Cartwright and Ray [1990] first published the sampling phase of adjacent ground tracks and showed how tidal errors can have a wavelike shape. Jacobs et al. [1992] showed that the aliased signals produced by altimetric sampling of tides appear as propagating waves and can satisfy the dispersion relation of Rossby waves. It was noted that the effect of aliasing on ascending and descending tracks will be different. Schlax and Chelton [1994] looked at the effect of tidal aliasing on timelongitude plots and showed that the wavelength of the

\footnotetext{
${ }^{1}$ Now at the Deptartment of Civil and Environmental Engineering and Geodetic Science, The Ohio State University, Columbus.
}

Copyright 1998 by the American Geophysical Union.

Paper number 97JC02175.

0148-0227/98/97JC-02175\$09.00 propagating waves is changed from that imposed by the sampling phase because adjacent altimeter tracks are not sampled at the same time. Such plots are very useful in detecting propagation in altimeter data. However, when considering only the east-west component of propagation as Schlax and Chelton did, the effect of aliasing is the same on both ascending and descending tracks. If the north-south component of propagation is considered, the direction of propagation for ascending tracks is different than that of descending tracks, and this can be used to help separate aliased from unaliased signals. Furthermore, all previous work has considered aliasing of deep water tides with long enough length scale so that it may be treated as having no spatial variation.

Here we review satellite aliasing characteristics and extend the work to aliasing of phenomena with shorter length scales and consider non-east-west propagation. The work presented here differs from previous work, including that of Schlax and Chelton, in three respects. We consider the aliasing of phenomena with finite wavelengths, aliasing of nontidal phenomena, and the northsouth as well as the east-west components of propagation of the aliases.

The manner in which altimetric satellites sample the ocean is governed by the fact that the ocean is not sampled as a snapshot, but rather as a sequence of individual tracks laid down from turning latitude to turning latitude. It is important to note, however, that the effects of measuring a given phenomena will be different for satellites in different orbits, and in particular can be radically different even for satellites with the same repeat period but different inclinations (and hence different ground tracks and ground track sequences). Thus, in addition to the time between repeats which is important for traditional aliasing, the time between adjacent tracks becomes important. 
The spatial distortion introduced by altimetric sampling means that measured phenomena can satisfy different dispersion relations than the underlying phenomena. Because of this it is possible for some aliased phenomena to satisfy the dispersion relation of other nonaliased oceanographic phenomena (or of other aliased phenomena) in some parts of the world. For example, with Geosat the aliased M2 tide satisfies the dispersion relation of annual Rossby waves [Jacobs et al., 1992]. More examples will be developed later in this paper. Thus, in addition to having aliased phenomena sometimes exhibit unexpected wavelengths, it is possible that in some parts of the world, analysis for one type of oceanic phenomenon will incorporate energy from another type of phenomenon (possibly not oceanographic in origin), even when using knowledge of ocean dynamics in the analysis.

The purpose of this paper therefore is to discuss the spatial as well as temporal characteristics of aliased signals, using the T/P, Geosat, and ERS orbits as examples. These results will be used to illustrate some of the possible confusions that can arise and the geographical locations for which the measured dispersion relations of different phenomena coincide.

When considering only temporal sampling it is usual to interpret an unknown sampled signal as the lowest possible frequency that could have created the observed measurements. In fact, it is generally part of experiment design to sample finely enough that only the lowest frequency signal represents a physically possible unknown. With altimetric data this is unrealistic. Adequate sampling in both space and time to resolve all oceanographic phenomena would require an unrealistic number of satellites [see, e.g., Bernstein et al., 1979; Chase and Mundt, 1989].

Unlike tidal errors, mesoscale variations occur at a wide range of frequencies and wavelengths and are not well known a priori. Thus the part of the mesoscale signal that is above the Nyquist frequency will alias to an entire range of frequencies. This will be true for many nontidal oceanographic phenomena, especially in shallow water. Note that the problem occurs for all orbits, although the aliased energy should be larger for ERS than Geosat and larger for Geosat than T/P because of their longer repeat periods. Mesoscale variations are largely unknown a priori, and regions of large mesoscale energy such as the Gulf Stream and Kuroshio extensions, the Antarctic Circumpolar Current, the Aghullas Retroflection, and the Brazil Current will all be problem areas for analysis. Along-track tidal solutions include mesoscale energy [Tierney et al., 1998] although combination of ascending and descending data together removes much of the energy.

This occurs because if ascending and descending track data are combined in a tidal solution at crossover points, then the aliasing problem is changed because the ascending and descending tracks are staggered in time. When this is done, much of the mesoscale contribution disappears. In the case of deep water tidal solutions produced thus far, this has been done either explicitly [Matsumoto et al., 1995] or implicitly through largescale binning and smoothing [see, e.g., Desai and Wahr, 1995]. In the future, when altimetric data is used to investigate tides to finer scales in shallow water or over bathymetric features, care must be taken to avoid incorporation of mesoscale energy into tidal solutions. The following discussion is divided into four sections: Atimetric aliasing, examples of aliasing, a discussion, and conclusions.

\section{Altimetric Aliasing}

\subsection{Two-Dimensional Aliasing}

Temporal aliasing and aliasing on regularly spaced grids sampled simultaneously is well understood. However, when sampling at neighboring ground tracks is lagged by an amount $\Delta t$ (where $\Delta t$ is considered to be the smallest-magnitude representation of the lag), then the situation becomes more complicated. This situation is well described by Schlax and Chelton [1994] and is directly applicable to time-longitude plots used to look for east-west propagating waves. For a phenomena of frequency $f$ there will be an expected lag in phase between neighboring grid points of $2 \pi f \Delta t$. Thus sampling of a spatially homogeneous signal will produce results that are not obviously spatially homogeneous, even when there is no temporal aliasing. For aliased phenomena the expected phase lag will be interpreted as $2 \pi\left[\frac{(\Delta t)}{(T)}-p\right]$, where $p$ is any integer. Hence all spatially homogeneous solutions at the principal alias frequency will appear to be propagating since the eye sees propagation and time-longitude plots will show propagating solutions. Schlax and Chelton designated the wavelength for $p=0$ to be $\lambda_{0}$, for $p=1$ to be $\lambda_{1}$, etc. In practice, only $\lambda_{0}$ and either $\lambda_{-1}$ or $\lambda_{1}$ (depending if $\Delta t$ is positive or negative) will be greater than the grid spacing (with either $\lambda_{-1}$ or $\lambda_{1}$ close to the grid spacing). These two aliases represent the principal westward propagating and eastward propagating aliases. Since we are ignoring subgrid solutions, these will be the only two considered. The value of $\lambda_{0}$ will always be greater than $\lambda_{-1}$ or $\lambda_{1}$. Schlax and Chelton recommend filtering spatially near the Nyquist to remove the second alias. However, there are scientifically interesting phenomena at or below the spatial Nyquist, such as will occur in coastal oceanography or with high-frequency waves or the surface expression of internal waves in deep water. Thus, whenever the spatial resolution of altimeter measurements is being pushed, this second alias must be considered. Like Schlax and Chelton we only consider the solutions at the principal alias frequency.

Consider an unaliased sinusoid of period $T$ and wavelength $X$. When sampling neighboring grid points at staggered times, the following signals will be identical at the sampled times and locations 


$$
\begin{aligned}
f\left(x_{k}, t_{j}+k \Delta t\right)= & \sin \left[2 \pi\left(\frac{1}{T}+\frac{n}{\Delta t_{0}}\right)\left(t_{j}+k \Delta t\right)\right. \\
& \left.+2 \pi\left(\frac{1}{X}-\frac{n \Delta t}{\Delta t_{0} \Delta x_{0}}+\frac{p}{\Delta x_{0}}\right) x_{k}\right]
\end{aligned}
$$

for $-\infty \leq n \leq \infty$ and $-\infty \leq p \leq \infty$. Note that although the temporal aliasing along a track is the same as when there is no time lag between neighboring grid points, the spatial wavelength is modified when the signal has been temporally aliased ( $n$ nonzero). Note that the integer $p$ introduced by Schlax and Chelton [1994] is simply the effect of spatial aliasing (because of the staggered measurement times at grid points one of the spatial aliases will in general have a wavelength longer than the grid spacing).

Since different grid points are sampled at different times, the principle alias of a spatially homogeneous signal is now considered to be the lowest wavenumber propagating solution. This occurs because it is no longer immediately obvious that the homogeneous signal is a solution. The eye and techniques seeking propagation pick out propagation.

\subsection{Three-Dimensional Aliasing}

Altimeter data consists of ascending and descending ground tracks, each of which is equally spaced in an east-west direction. Schlax and Chelton, [1994] consider the case of time-longitude plots, which is equivalent to the two-dimensional aliasing problem. A similar case can be constructed by considering ground track points at one longitude. The only difference is that now the grid points are not equally spaced but get closer together at higher latitudes. Thus, in general, the direction of propagation of the alias is not east-west but at an angle. If the adjacent track is to the north for ascending tracks, then it will be to the south for descending tracks and vice versa. Therefore, if the direction of propagation of the alias is to the northwest for ascending tracks, it will be to the southwest for descending tracks and the angle to the equator will be approximately the same. The effect that this has on sampling oceanographic phenomena can be illustrated by looking at three special cases: a spatially homogeneous signal, a plane wave traveling cross track, and a plane wave traveling along track.

\subsection{Case 1: Sampling a Spatially Homogeneous Signal}

This is the case considered by Jacobs et al. [1992] and Schlax and Chelton [1994]. Suppose that the underlying phenomenon is composed solely of a sinusoidal variation in time but constant phase horizontally, i.e., $a=\sin (2 \pi f t)$, where $f$ is the frequency of the underlying phenomenon. This situation corresponds roughly to the aliasing of very broad scale phenomena such as deep water tides where the distance between adjacent ground tracks is much less than the wavelength of the measured phenomena. Because the amount of time for a satellite to measure a complete ascending or descending track ( $\sim 50 \mathrm{~min}$ for the Geosat) is small compared to the timescales of typical oceanographic variations, adjacent ground tracks may be considered to be sampled at a constant time apart for purposes of this discussion. Thus, if at a given point on a given ground track the underlying phenomena is sampled at times $t_{j}$, the equivalent point on the next track to the east will be sampled at times $t_{j}+\Delta t$. In other words, the measurements at the given point will be $\sin \left(2 \pi f t_{j}\right)$ while the measurements on the track to the east will be $\sin \left[2 \pi f\left(t_{j}+\Delta t\right)\right]$. If one adopts a local coordinate system aligned with the ground tracks such that $y$ is aligned with the ground tracks while $x$ is normal to the ground tracks, then given a ground track separation of $d$, the measurements at the adjacent track can be considered as analagous to the staggered two-dimensional case above. Because ground tracks effectively represent the shape of wave crests (the measured phenomenon is constant along a track), the direction of propagation will be normal to the ground tracks at an angle $\beta$ to the meridian and thus be a function of latitude and dependent on whether one is considering ascending or descending ground tracks.

The direction of propagation of the measured wave may be calculated simply by considering that the normal to the ground track is perpendicular to the velocity vector relative to the Earth at the measured location. Consider the angle $\gamma$ between a satellite ground track and north (i.e., a meridian). The angle $\gamma$ is the angle between the meridian and the satellite velocity vector seen by an observer fixed to the Earth. This angle is found by subtracting the Earth's surface rotational velocity vector from the satellite velocity vector projected on the Earth's surface. For a circular orbit the angle $\gamma$ is given by [Parke et al. (1987)] as

$$
\gamma=\tan ^{-1} \operatorname{abs}\left(\frac{V_{S} \sin \alpha \pm V_{E} \cos \phi}{V_{S} \cos \alpha}\right)
$$

where the two terms in the numerator are added for retrograde orbits and subtracted for prograde orbits, $V_{S}$ is the spacecraft velocity

$$
V_{S}=\frac{R_{E}}{a}\left(\frac{\mu_{E}}{a}\right)^{\frac{1}{2}}
$$

and

$$
\sin \alpha=\operatorname{abs}\left(\frac{\cos i}{\cos \phi}\right)
$$

where $i$ is the inclination of the satellite orbit and $\phi$ is latitude. The quantity $V_{E}$ is the equatorial rotation velocity of the Earth given by $R_{E} \omega_{E}$.

The perpendicular distance between ground tracks $d$ is approximately given by

$$
d=D \cos (\gamma)
$$

where $D$ is the east-west spacing between adjacent ground tracks. For T/P, D is $312 \mathrm{~km}$ while for Geosat, 


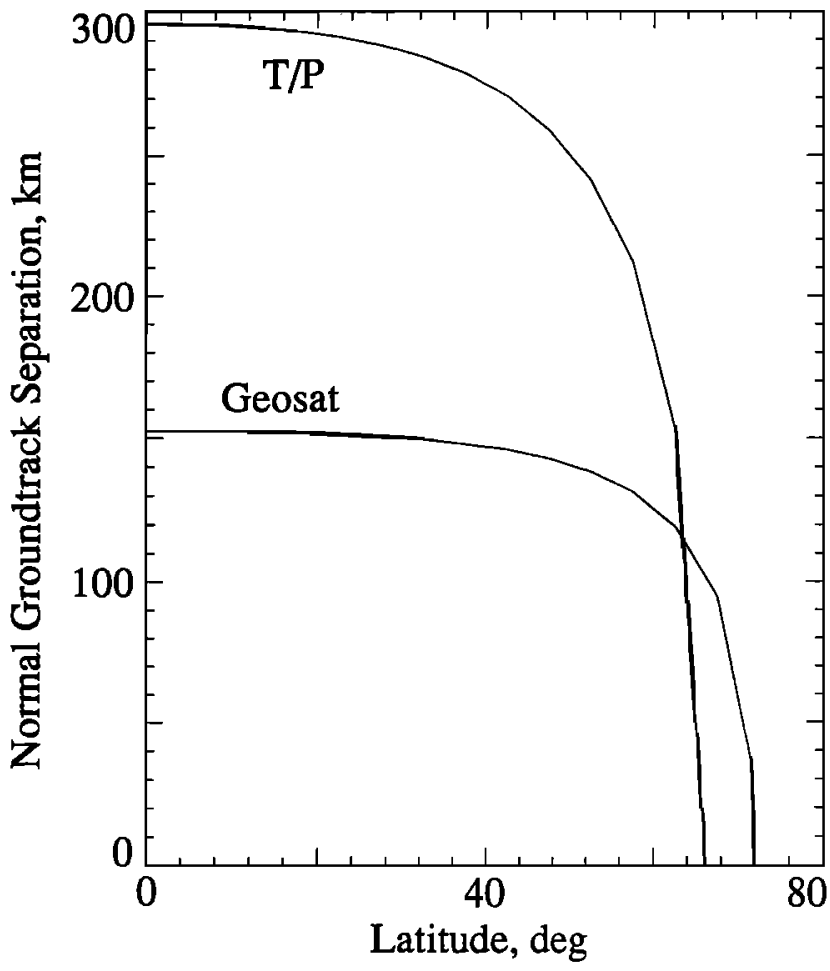

Figure 1. Ground track spacing as a function of latitude.

$D$ is $162 \mathrm{~km}$. The vertical distance $L$ between tracks is approximately

$$
L=D \cot (\gamma)
$$

Figure 1 shows the variation in $d$ with latitude for Geosat and T/P. As one follows a ground track towards the turning latitude the direction of propagation of the apparent wave tends toward north-south, and the wavelength tends toward zero.

The direction of propagation of the apparent wave will be normal to the ground track and may be either along the easterly normal or along the westerly normal. Because the wavelength of the aliased phenomena varies with latitude, it is most proper to report wavelengths in tracks.

\subsection{Case 2: Sampling a Plane Wave Traveling Cross Track}

Assume that the underlying phenomenon is a plane wave traveling in a cross-track direction with wavelength $\lambda_{u}$. When $\lambda_{u}<2 d$, the phenomenon will be aliased spatially. For this example, only $\lambda_{u}>2 d$ will be considered. The wavenumber of the underlying phenomenon is $\kappa_{u}=2 \pi / \lambda_{u}$.

The phase at an adjacent track is determined by the contribution from the altimeter sampling as discussed in the section on aliasing spatially homogeneous signals plus the contribution from the underlying phenomenon itself. Thus the measured wavenumber $\kappa$ is given by $\kappa=\kappa_{u}+\kappa_{s}$, where $\kappa_{s}$ is the wavenumber found by sampling a spatially homogeneous signal. This could be either the principal easterly or westerly alias. Similarly, $\lambda_{s}$ is the corresponding wavelength found by sampling a spatially homogeneous signal. Therefore the measured wavelength $\lambda$ is given by

$$
\frac{\lambda_{u} \lambda_{s}}{\lambda_{u}+\lambda_{s}}
$$

The direction of propagation will be determined by the signs of $\kappa$ and $\Delta t$.

Since $\lim _{\lambda_{u} \rightarrow \infty} \kappa_{u}=0$, then $\lim _{\lambda_{u} \rightarrow \infty} \kappa=\kappa_{g}$ and the situation is case 1 as before. Note that if $\kappa_{g}$ and $\kappa_{u}$ are of opposite signs, then the direction of propagation will switch as $\lambda_{u}$ becomes larger. Figure 2 shows an example of the change in propagation of the measured wave as $\lambda_{u}$ varies for a given satellite $(T / P$ in this case) and hence a fixed $\lambda_{s}$. Note that when $\lambda_{u}$ and $\lambda_{s}$ are equal and of opposite sign, the apparent wavelength of the measured phenomena is infinite. Thus propagating phenomena sampled by a radar altimeter can appear nonpropagating as well as the other way around.

\subsection{Case 3: Sampling a Plane Wave Traveling Along Track}

Assume that the underlying phenomenon is a plane wave traveling in an along-track direction. In this case the vector wavenumber of the observed alias will be given by the vector sum of $\kappa_{u}$ and $\kappa_{s}$ (which are per-

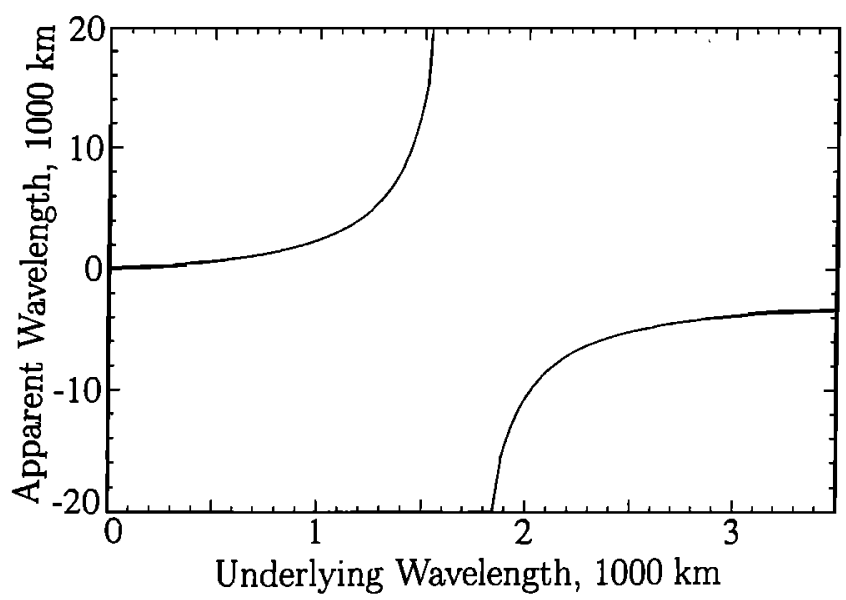

Figure 2. Apparent wavelength of a measured plane wave traveling in a cross-track direction as a function of the wavelength of the underlying wave for a given satellite (TOPEX/POSEIDON with equatorial ground track spacing in this case) and hence a fixed $\lambda_{s}$. Note that when the wavelength of the underlying wave is small, the underlying wave dominates the wavelength of the apparent wave, while when the wavelength of the underlying wave is large, the sampling of the satellite dominates. When $\lambda_{u}$ and $\lambda_{s}$ are equal and of opposite sign as in the case shown here, the apparent wavelength of the measured phenomenon is infinite, and a propagating phenomenon sampled by a radar altimeter can appear nonpropagating as well as the other way around. 


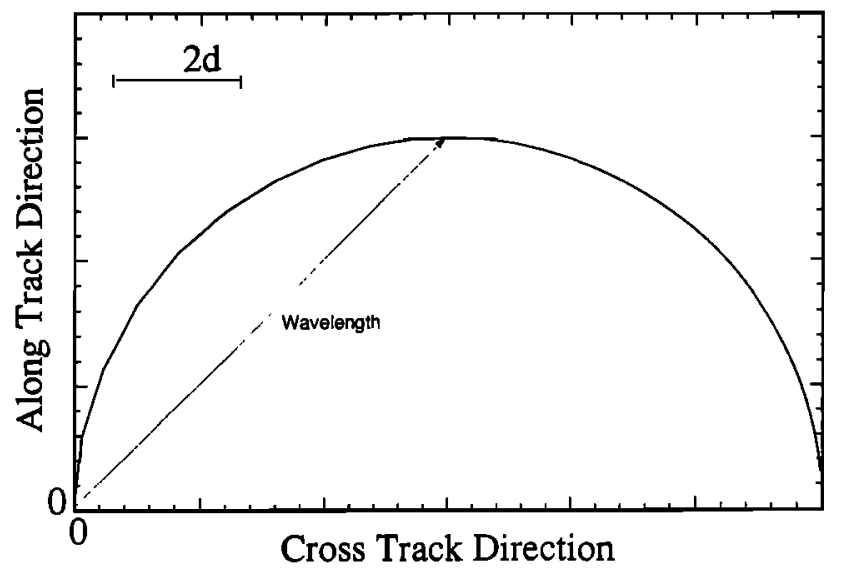

Figure 3. The apparent wavelength and direction of propagation of the apparent wavelength of a measured plane wave traveling in an ] along-track direction as a function of the wavelength of the underlying wave (TOPEX/POSEIDON in this case). Each point on the displayed curve represents the results of sampling one underlying wavelenth. If a vector is drawn from the origin to a point on the displayed curve, then the angle from the vertical axis represents the angle of propagation of the measured wave with respect to the alongtrack direction while the length of the vector represents the wavelength of the apparent wave. The distance bar represents twice the ground track spacing at the latitude of interest. The left end of the curve represents the case where the underlying wavelength tends to zero, while the right end of the curve represents the case where the underlying wavelength tends to infinity. Note that when the underlying wavelength tends to be small, the propagation of the apparent wave tends to be along track, while when the underlying wavelength tends to be large, the propagation tends to be cross track.

pendicular to each other). Thus the direction of propagation of the aliased signal will be at an angle $\nu$ to the ground track given by

$$
\nu=\tan ^{-1}\left[\frac{\lambda_{u}}{\lambda_{s}}\right]
$$

Note that at the extremes, $\lim _{\lambda_{u} \rightarrow 0} \nu=0$, and $\lim _{\lambda_{u} \rightarrow \infty} \nu= \pm \pi / 2$. Thus the $\lim _{\lambda_{u} \rightarrow \infty}$ represents case 1 as might be expected. As the wavelength of the underlying phenomena trends from zero to infinity, the direction of propagation of the aliased wave rotates from the along-track to the cross-track direction. Figure 3 shows an example of the change in propagation of the measured phenomena as $\lambda_{u}$ varies.

\subsection{Sampling an Arbitrary Plane Wave}

Any plane wave may be considered as being composed of along-track and cross track components, and thus its aliased behavior can be discussed in terms of cases 2 and 3 above. An arbitrary wave will have a direction of propagation that rotates to one of the normals to the ground track as $\lambda_{u}$ tends to infinity. If one considers a wave for which the cross-track component of $\kappa_{z}$ and $\kappa_{u}$ are of opposite signs, then this rotation will cover an angle greater than $90^{\circ}$. Thus it is possible for a plane wave with finite wavelength to have an alias that propagates at right angles to the underlying wave.

\section{Sampling Oceanographic Phenomena}

It should be noted that the problems of sampling discussed in this paper affect all phenomena that appear in altimeter data, whether oceanographic, atmospheric, related to orbit determination, instrumental, or from other causes. It is the intent of this paper to limit the discussion to oceanographic signals, but similar problems caused by other sources will occur in the analysis of altimeter data. These may well be important in a given analysis.

Because of the complexity of the ocean, its stratification and topography it would be a daunting task to discuss the result of sampling all known oceanographic phenomena. It is the intent here to discuss some examples that are either of known importance or thought to be illustrative. The cases chosen here are tides, Rossby waves, and equatorially trapped waves.

\subsection{Tides}

Tidal variations are pervasive in the deep ocean. Even when the best contemporary solutions are applied to altimeter data, there are still significant residual errors [Shum et al., 1997]. As discussed below, the result of sampling the tides involves a wide variety of frequencies and wavenumbers; often radically different from the original tidal signal.

The temporal aliasing of tides at a single location has been discussed extensively in other publications [see, e.g., Parke et al., 1987; Cartwright and Ray, 1990]. The east-west spatial aliasing of the six largest tidal constituents is discussed by Schlax and Chelton [1994]. In regions where the amplitude of a given tidal constituent is locally large it is often the case that the phase of the tide is slowly varying in space (such locations are often called antiamphidromes). In these cases the alias of the tide behaves much like case 1 , the spatially homogeneous case of section 3 . Thus it is meaningful to discuss the sampling of an idealized tide with no spatial variation. However, it must be noted that aliased wavelengths will differ from the homogeneous case depending on the actual wavelength and direction of propagation of the tide. Tables 1 and 2 give for $T / P$ the principal alias period, wavelength of the principal east and west aliases for the limit $\lambda_{u}$ tends to infinity, and east-west phase speed for every tidal constituent for which the equilibrium forcing amplitude is $>0.1 \mathrm{~cm}$. Each of these constituents will be expected somewhere in deep water to have an amplitude approaching or exceeding $1 \mathrm{~cm}$. Coastal amplitudes could be greater. Tables 3 and 4 give similar results for Geosat, while Tables 5 and 6 are for ERS. When the apparent wavelength listed in Ta- 
Table 1. TOPEX/POSEIDON Data for Selected Terdiurnal and Semidiurnal Tidal Constituents

\begin{tabular}{|c|c|c|c|c|c|c|}
\hline \multirow[b]{2}{*}{ Period, days } & \multirow[b]{2}{*}{ Symbol } & \multirow[b]{2}{*}{ Alias Period, days } & \multicolumn{2}{|c|}{ East } & \multicolumn{2}{|c|}{ West } \\
\hline & & & Wavelength, km & Velocity, $\mathrm{cm} / \mathrm{s}$ & Wavelength, km & Velocity, $\mathrm{cm} / \mathrm{s}$ \\
\hline 8.2804008 & M3 & 38.05 & 569.90 & 17.33 & -620.45 & -18.87 \\
\hline 12.4206012 & M2 & 62.12 & 943.13 & 17.57 & -433.63 & -8.08 \\
\hline 12.0000000 & S2 & 58.73 & 336.33 & 6.63 & -2543.63 & -50.13 \\
\hline 12.6583482 & N2 & 49.51 & 525.58 & 12.29 & -683.17 & -15.97 \\
\hline 11.9672348 & $\mathbf{K 2}$ & 86.57 & 324.40 & 4.34 & -3524.15 & -47.12 \\
\hline 12.6260044 & $\nu 2$ & 65.20 & 500.06 & 8.88 & -731.72 & -12.99 \\
\hline 12.1916202 & L2 & 20.63 & 426.37 & 23.92 & -979.37 & -54.93 \\
\hline 12.0164492 & $\mathbf{T 2}$ & 50.60 & 342.63 & 7.84 & -2233.01 & -51.08 \\
\hline 12.9053745 & 2N2 & 22.54 & 483.66 & 24.84 & -769.91 & -39.53 \\
\hline 12.6604146 & $\cdots$ & 48.77 & 527.27 & 12.51 & -680.34 & -16.14 \\
\hline 12.8717576 & $\mu 2$ & 20.32 & 483.66 & 27.55 & -769.91 & -43.86 \\
\hline 12.1897040 & $\cdots$ & 20.77 & 425.25 & 23.70 & -985.34 & -54.91 \\
\hline 12.2217742 & $\lambda 2$ & 21.04 & 943.13 & 51.89 & -433.63 & -23.86 \\
\hline 11.7545217 & KJ2 & 40.42 & 18860.62 & 540.07 & -301.81 & -8.64 \\
\hline 12.9075223 & $\cdots$ & 22.70 & 483.66 & 24.66 & -769.91 & -39.26 \\
\hline
\end{tabular}

bles 1-6 is small, the resulting wavelength will appear throughout much of the deep water. When the apparent wavelength is large, the underlying wavelength of the tide will dominate, and the sampled tide will appear as if it were simply aliased.

In real analyses, methods for removing orbit error will transfer energy from the regions where there is tidal error to those that have no error. However, the patterns will be characteristic for a given tidal frequency and can often be readily recognized.

\subsection{Rossby Waves}

The apparent frequency and wavenumber of the M2 tidal alias for Geosat have been shown to satisfy the dispersion relation of annual first-mode baroclinic Rossby waves at midlatitudes in the Pacific [Jacobs, et al., 1992], where the dimensionful dispersion relation for a first-mode baroclinic Rossby wave is given by

$$
\sigma=\frac{-\beta \kappa}{\kappa^{2}+\lambda^{2}+\left(\frac{1}{R_{1}}\right)^{2}}
$$

Table 2. TOPEX/POSEIDON Data for Selected Diurnal and Long-Period Tidal Constituents

\begin{tabular}{|c|c|c|c|c|c|c|}
\hline \multirow[b]{2}{*}{ Period, days } & \multirow[b]{2}{*}{ Symbol } & \multirow[b]{2}{*}{ Alias Period, days } & \multicolumn{2}{|c|}{ East } & \multicolumn{2}{|c|}{ West } \\
\hline & & & Wavelength, km & Velocity, $\mathrm{cm} / \mathrm{s}$ & Wavelength, km & Velocity, $\mathrm{cm} / \mathrm{s}$ \\
\hline 23.9344697 & K1 & 173.14 & 310.12 & 2.07 & -7048.29 & -47.12 \\
\hline 25.8193417 & 01 & 45.72 & 967.32 & 24.49 & -428.70 & -10.85 \\
\hline 24.0658902 & P1 & 88.88 & 321.01 & 4.18 & -3979.94 & -51.83 \\
\hline 26.8683567 & Q1 & 69.36 & 489.09 & 8.16 & -756.55 & -12.62 \\
\hline 24.8332484 & M1 & 23.77 & 400.06 & 19.48 & -1153.64 & -56.17 \\
\hline 23.0984768 & J1 & 32.77 & 37721.24 & 1332.30 & -299.41 & -10.58 \\
\hline 22.3060742 & OO1 & 129.92 & 3193.95 & 123.56 & -327.51 & -12.67 \\
\hline 26.7230533 & $\rho 1$ & 104.59 & 466.93 & 5.17 & -816.48 & -9.04 \\
\hline 23.0915993 & $\ldots$ & 32.44 & 37721.24 & 1345.81 & -299.41 & -10.68 \\
\hline 26.8776683 & $\ldots$ & 67.90 & 490.57 & 8.36 & -753.03 & -12.84 \\
\hline 24.1321400 & $\pi 1$ & 71.48 & 326.75 & 5.29 & -3268.53 & -52.92 \\
\hline 28.0062225 & 2Q1 & 19.94 & 489.94 & 28.43 & -754.51 & -43.79 \\
\hline 23.8044765 & $\phi 1$ & 3336.79 & 299.95 & 0.10 & -30772.38 & -10.67 \\
\hline 27.8483876 & $\sigma 1$ & 21.81 & 705.86 & 37.46 & -512.90 & -27.22 \\
\hline 25.8107490 & $\cdots$ & 45.08 & 967.32 & 24.84 & -428.70 & -11.01 \\
\hline 327.858969 & Mf & 36.17 & 2197.94 & 70.34 & -343.47 & -10.99 \\
\hline 661.309205 & $\mathrm{Mm}$ & 27.55 & 2197.94 & 92.32 & -343.47 & -14.43 \\
\hline 4382.90521 & Ssa & 182.62 & 2197.94 & 13.93 & -343.47 & -2.18 \\
\hline 655.717998 & $\ldots$ & 27.32 & 2197.94 & 93.11 & -343.47 & -14.55 \\
\hline 219.190386 & $\ldots$ & 115.71 & 423.88 & 4.24 & -992.78 & -9.93 \\
\hline
\end{tabular}


Table 3. Geosat Data for Selected Terdiurnal and Semidiurnal Tidal Constituents

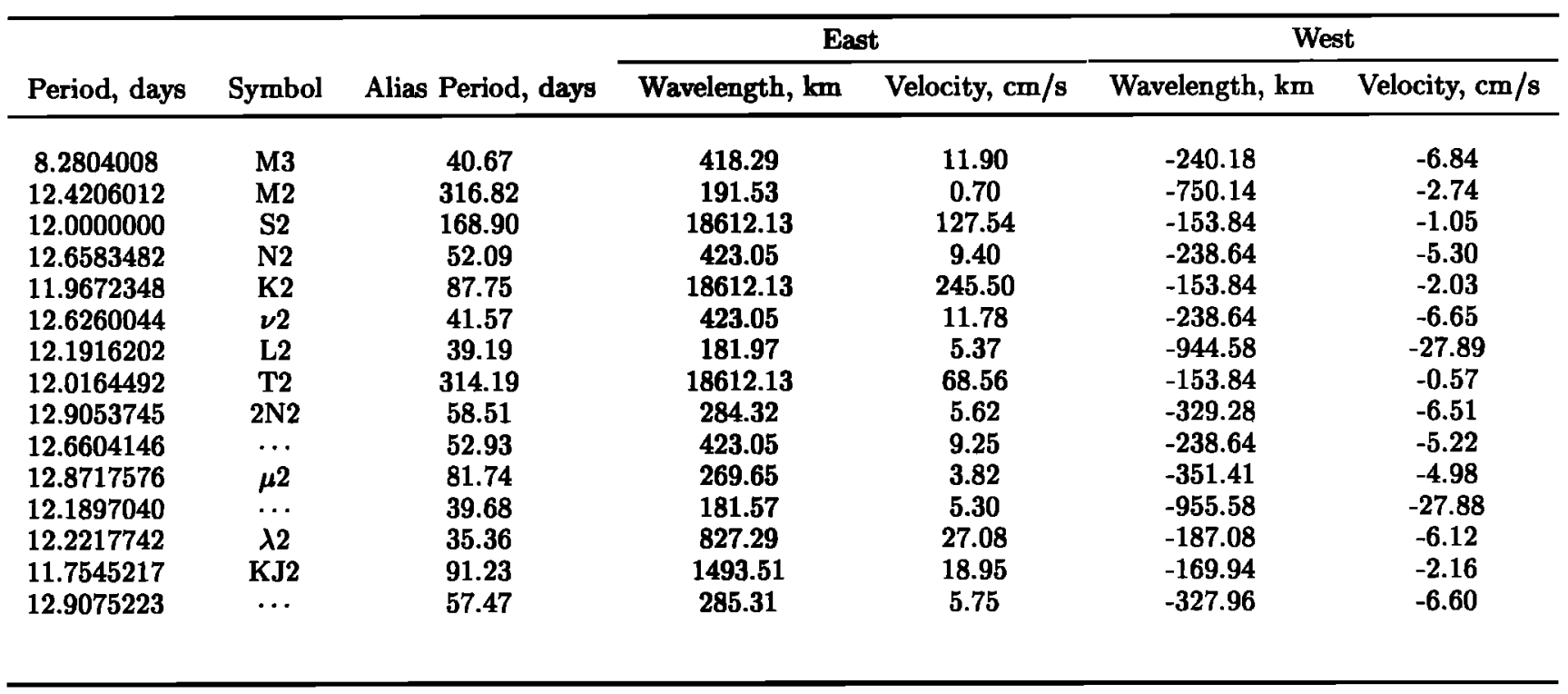

with $\sigma$, the frequency of the Rossby wave; $\kappa$, the eastwest wavenumber; $\lambda$, the north-south wavenumber, $\beta$ the coriolis parameter; and $R_{1}$, the internal rossby radii. For the calculations shown here, Rossby radii from Emery et al. [1984] were used. Note that the wavelength of a given frequency Rossby wave is dependent on the direction of propagation, the latitude, and the vertical structure of the ocean.

In determining the latitude where the alias of a given tide satisfies the first-mode baroclinic Rossby wave dispersion relation, it is assumed that the tidal signal behaves approximately as if it were spatially homogeneous. This will be approximately true in deep water, but the approximation will suffer in shallow water. The direction of propagation of the aliased tide is taken to be normal to the altimeter ground tracks. Because the wavelengths of Rossby waves depend on the vertical structure of the ocean, the latitude where the tide satisfies the Rossby wave dispersion relation is a function of longitude.

Figure 4 shows a map of the latitude in the North Pacific where the apparent frequency and wavenumber of

Table 4. Geosat Data for Selected Diurnal and Long-Period Tidal Constituents

\begin{tabular}{|c|c|c|c|c|c|c|}
\hline \multirow[b]{2}{*}{ Period, days } & \multirow[b]{2}{*}{ Symbol } & \multirow[b]{2}{*}{ Alias Period, days } & \multicolumn{2}{|c|}{ East } & \multicolumn{2}{|c|}{ West } \\
\hline & & & Wavelength, km & Velocity, $\mathrm{cm} / \mathrm{s}$ & Wavelength, km & Velocity, $\mathrm{cm} / \mathrm{s}$ \\
\hline 23.9344697 & $\mathbf{K} 1$ & 175.49 & 37224.26 & 245.50 & -153.20 & -1.01 \\
\hline 25.8193417 & $\mathrm{O} 1$ & 112.94 & 199.06 & 2.04 & -653.31 & -6.70 \\
\hline 24.0658902 & P1 & 4496.55 & 37224.26 & 9.58 & -153.20 & -0.04 \\
\hline 26.8683567 & Q1 & 74.06 & 427.91 & 6.69 & -237.12 & -3.71 \\
\hline 24.8332484 & M1 & 35.64 & 846.10 & 27.47 & -186.14 & -6.04 \\
\hline 23.0984768 & J1 & 60.03 & 2118.70 & 40.85 & -164.42 & -3.17 \\
\hline 22.3060742 & OO1 & 49.38 & 184.30 & 4.32 & -886.39 & -20.78 \\
\hline 26.7230533 & $\rho 1$ & 54.47 & 427.91 & 9.09 & -237.12 & -5.04 \\
\hline 23.0915993 & $\cdots$ & 61.16 & 2065.36 & 39.08 & -164.75 & -3.12 \\
\hline 26.8776683 & $\cdots$ & 75.79 & 427.91 & 6.53 & -237.12 & -3.62 \\
\hline 24.1321400 & $\pi 1$ & 397.55 & 155.56 & 0.45 & -7940.31 & -23.12 \\
\hline 28.0062225 & 2Q1 & 43.88 & 301.24 & 7.95 & -309.16 & -8.15 \\
\hline 23.8044765 & $\phi 1$ & 89.49 & 37224.26 & 481.42 & -153.20 & -1.98 \\
\hline 27.8483876 & $\sigma 1$ & 55.77 & 284.82 & 5.91 & -328.60 & -6.82 \\
\hline 25.8107490 & $\cdots$ & 117.03 & 198.58 & 1.96 & -658.56 & -6.51 \\
\hline 327.858969 & Mf & 68.72 & 185.22 & 3.12 & -865.77 & -14.58 \\
\hline 661.309205 & Mm & 44.73 & 3644.15 & 94.30 & -159.24 & -4.12 \\
\hline 4382.90521 & Ssa & 182.62 & 3644.15 & 23.10 & -159.24 & -1.01 \\
\hline 655.717998 & $\ldots$ & 45.35 & 3489.17 & 89.04 & -159.55 & -4.07 \\
\hline 219.190386 & $\ldots$ & 128.12 & 499.34 & 4.51 & -219.71 & -1.98 \\
\hline
\end{tabular}


Table 5. ERS (35 day) Data for Selected Terdiurnal and Sernidiurnal Tidal Constituents

\begin{tabular}{|c|c|c|c|c|c|c|}
\hline \multirow[b]{2}{*}{ Period, days } & \multirow[b]{2}{*}{ Symbol } & \multirow[b]{2}{*}{ Alias Period, days } & \multicolumn{2}{|c|}{ East } & \multicolumn{2}{|c|}{ West } \\
\hline & & & Wavelength, km & Velocity, cm/s & Wavelength, km & Velocity, $\mathrm{cm} / \mathrm{s}$ \\
\hline 8.2804008 & M3 & 78.76 & 93.62 & 1.38 & -471.41 & -6.93 \\
\hline 12.4206012 & M2 & 94.49 & 105.09 & 1.29 & -304.17 & -3.73 \\
\hline 12.0000000 & $\mathrm{S2}$ & $\infty$ & 78.42 & 0.96 & -19617.07 & -240.30 \\
\hline 12.6583482 & N2 & 97.44 & 93.84 & 1.11 & -465.81 & -5.53 \\
\hline 11.9672348 & $\mathrm{~K} 2$ & 182.62 & 19617.07 & 124.33 & -78.42 & -0.50 \\
\hline 12.6260044 & $\nu 2$ & 74.37 & 401.54 & 6.25 & -96.97 & -1.51 \\
\hline 12.1916202 & L2 & 349.25 & 174.61 & 0.58 & -141.32 & -0.47 \\
\hline 12.0164492 & T2 & 365.26 & 85.98 & 0.27 & $-\mathbf{8 5 2 . 8 7}$ & -2.70 \\
\hline 12.9053745 & 2N2 & 392.55 & 269.88 & 0.80 & -109.92 & -0.32 \\
\hline 12.6604146 & $\ldots$ & 100.42 & 93.84 & 1.08 & -465.81 & -5.37 \\
\hline 12.8717576 & $\mu 2$ & 135.06 & 269.88 & 2.31 & -109.92 & -0.94 \\
\hline 12.1897040 & $\cdots$ & 391.56 & 170.83 & 0.50 & -143.90 & -0.43 \\
\hline 12.2217742 & $\lambda 2$ & 129.53 & 267.56 & 2.39 & -110.30 & -0.99 \\
\hline 11.7545217 & KJ2 & 75.78 & 142.82 & 2.18 & -172.38 & -2.63 \\
\hline 12.9075223 & $\ldots$ & 446.83 & 269.88 & 0.70 & -109.92 & -0.28 \\
\hline
\end{tabular}

the principal westerly M2 tidal alias for Geosat satisfy the dispersion relation of first-mode baroclinic Rossby waves. Note the change in latitude near the coasts. In practice, the amount of data available is limited, and so tidal aliases that are close to satisfying the Rossby wave dispersion relation also cannot be separated from Rossby waves. Thus there is a latitude band where aliasing is a problem, rather than one single latitude. The dashed lines represent the limit of signals that can be separated based on frequency using 2 years of data. This represents an approximation to the problem lat- itudes, since signals that agree in frequency but differ slightly in wavelength also cannot be separated. Also, the real tide will behave differently than a spatially homogeneous tide and so will have a somewhat different wavelength. Figure 5 shows a similar plot for the North Atlantic.

Figures 6 and 7 show similar maps for the S2 tide in the North Pacific and North Atlantic for Geosat, while Figures 8 and 9 show the situation for $T / P$. Note that the latitudes where the $\mathrm{S} 2$ tidal alias satisfies the dispersion relation of first-mode baroclinic Rossby

Table 6. ERS (35 day) Data for Selected Diurnal and Long-Period Tidal Constituents

\begin{tabular}{|c|c|c|c|c|c|c|}
\hline \multirow[b]{2}{*}{ Period, days } & \multirow[b]{2}{*}{ Symbol } & \multirow[b]{2}{*}{ Alias Period, days } & \multicolumn{2}{|c|}{ East } & \multicolumn{2}{|c|}{ West } \\
\hline & & & Wavelength, km & Velocity, cm/s & Wavelength, km & Velocity, $\mathrm{cm} / \mathrm{s}$ \\
\hline 23.9344697 & $\mathbf{K} 1$ & 365.24 & 39234.14 & 124.33 & -78.26 & -0.25 \\
\hline 25.8193417 & O1 & 75.07 & 118.77 & 1.83 & -228.11 & -3.52 \\
\hline 24.0658902 & P1 & 365.24 & 85.79 & 0.27 & -871.78 & -2.76 \\
\hline 26.8683567 & Q1 & 132.81 & 94.06 & 0.82 & -460.35 & -4.01 \\
\hline 24.8332484 & M1 & 200.71 & 204.73 & 1.18 & -126.28 & -0.73 \\
\hline 23.0984768 & $\mathrm{~J} 1$ & 95.62 & 143.34 & 1.73 & -171.62 & -2.08 \\
\hline 22.3060742 & 001 & 102.31 & 1381.59 & 15.63 & -82.79 & -0.94 \\
\hline 26.7230533 & $\rho 1$ & 80.73 & 94.06 & 1.35 & -460.35 & -6.60 \\
\hline 23.0915993 & $\ldots$ & 92.87 & 143.34 & 1.79 & -171.62 & -2.14 \\
\hline 26.8776683 & $\ldots$ & $\mathbf{1 3 8 . 5 0}$ & 94.06 & 0.79 & -460.35 & -3.85 \\
\hline 24.1321400 & $\pi 1$ & 182.63 & 94.92 & 0.60 & -440.80 & -2.79 \\
\hline 28.0062225 & 2Q1 & 5250.93 & 110.55 & 0.02 & -266.10 & -0.06 \\
\hline 23.8044765 & $\phi 1$ & 121.75 & 39234.14 & 372.98 & -78.26 & -0.74 \\
\hline 27.8483876 & $\sigma 1$ & 214.30 & 271.74 & 1.47 & -109.61 & -0.59 \\
\hline 25.8107490 & $\cdots$ & 76.85 & 117.01 & 1.76 & -234.91 & -3.54 \\
\hline 327.858969 & Mf & 79.92 & 80.44 & 1.16 & -2685.98 & -38.90 \\
\hline 661.309205 & $\mathrm{Mm}$ & 129.53 & 143.86 & 1.29 & -170.88 & -1.53 \\
\hline 4382.90521 & Ssa & 182.62 & 143.86 & 0.91 & -170.88 & -1.08 \\
\hline 655.717998 & No & 124.54 & 143.86 & 1.34 & -170.88 & -1.59 \\
\hline 219.190386 & $\ldots$ & 208.69 & 115.71 & 0.64 & -240.34 & -1.33 \\
\hline
\end{tabular}




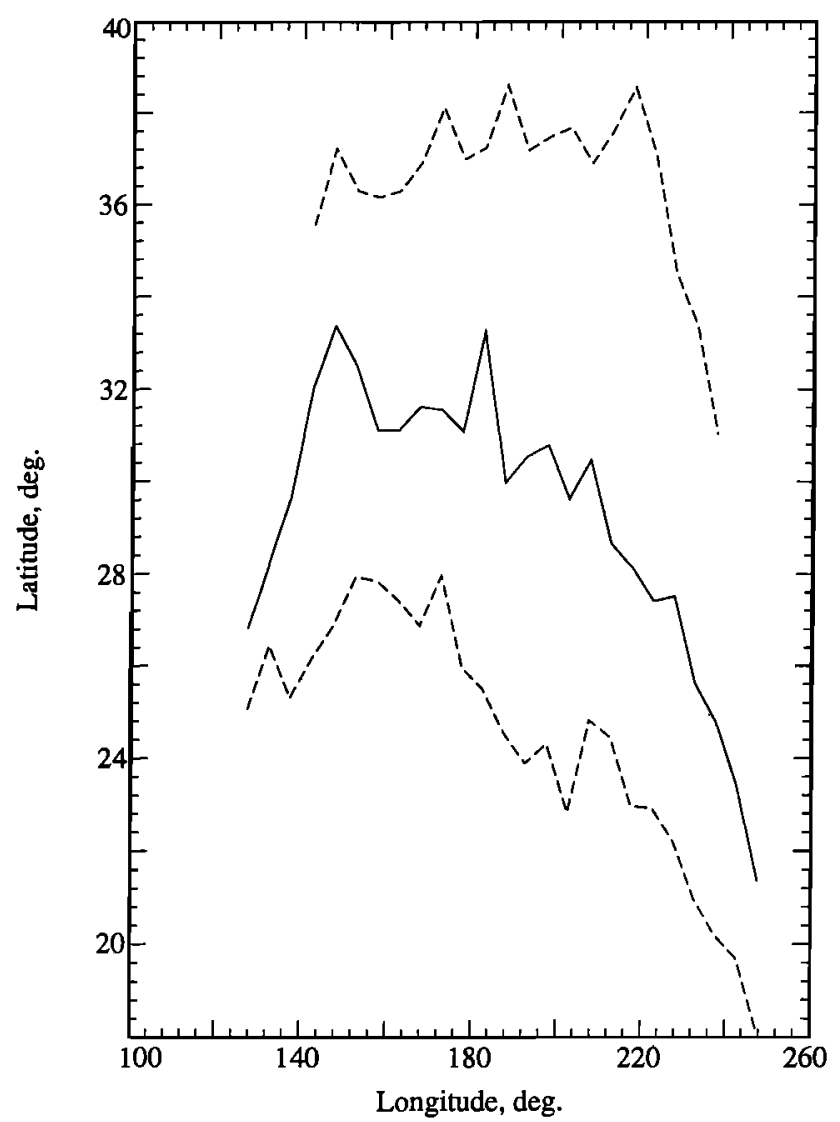

Figure 4. Map of the North Pacific showing the latitude where the apparent frequency and wavenumber of the aliased M2 tide for Geosat matches that of a first mode baroclinic Rossby wave. The solid line represents the latitude where there is an exact match between frequency and wavenumber while the dashed lines represent the latitudes where the two can be separated by frequency using 2 years of data.

waves are very similar for both $\mathrm{T} / \mathrm{P}$ and Geosat. Figures 10 and 11 show similar latitudes for ERS and the M2 tide.

It is important to note that when data from different satellites with different sampling problems are combined, it should be possible to create an analysis scheme that will allow separation of many signals. Care needs to be taken, however, that both missions have a common absolute reference so that artificial signals are not generated, and care needs to be taken when combining data from satellites with similar problems such as satellites in the T/P and Geosat orbits as described above.

\subsection{Equatorial Trapped Waves}

The equatorial region is one of many fast waves [Philander, 1990] that will be aliased with both Geosat and $\mathrm{T} / \mathrm{P}$ data. Two examples will be explored here: the near-4-day internal gravity wave mode [see, e.g., Groves and Miyata 1967; Miyata and Groves 1968; Wunsch and Gill, 1976] and equatorial Kelvin waves.

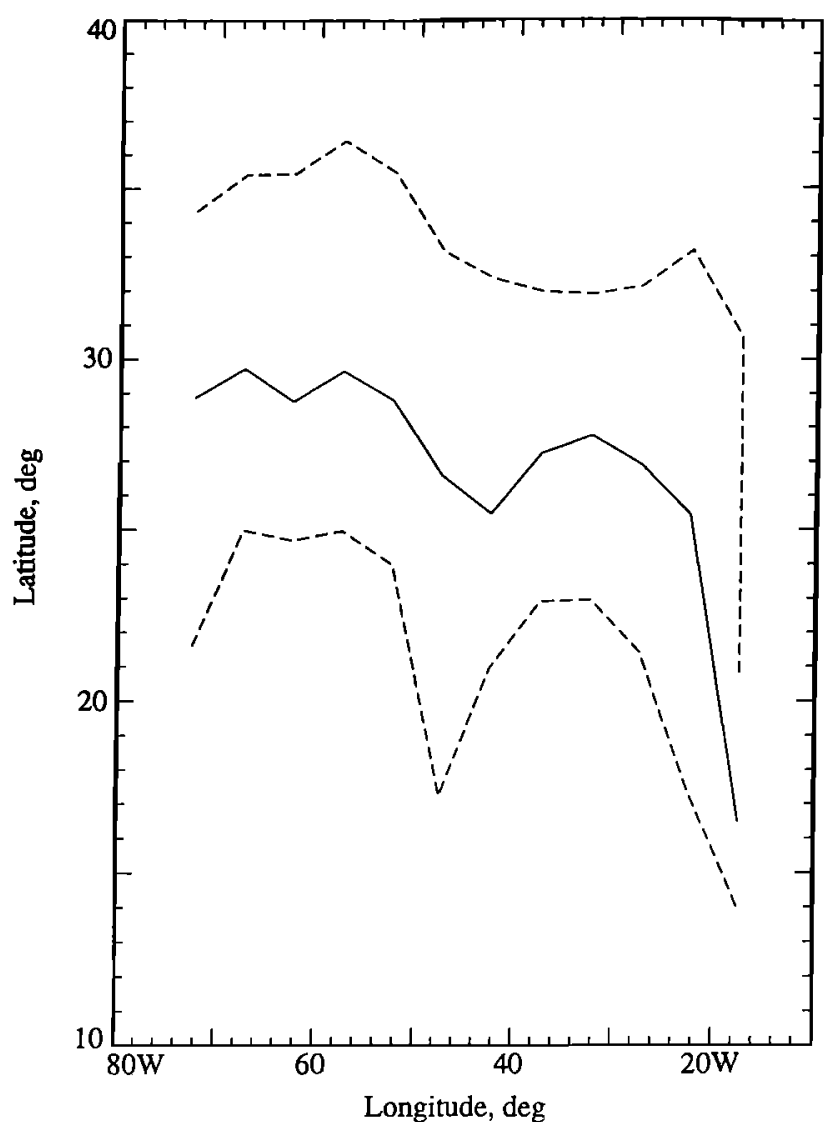

Figure 5. The same as Figure 4, except for the North Atlantic.

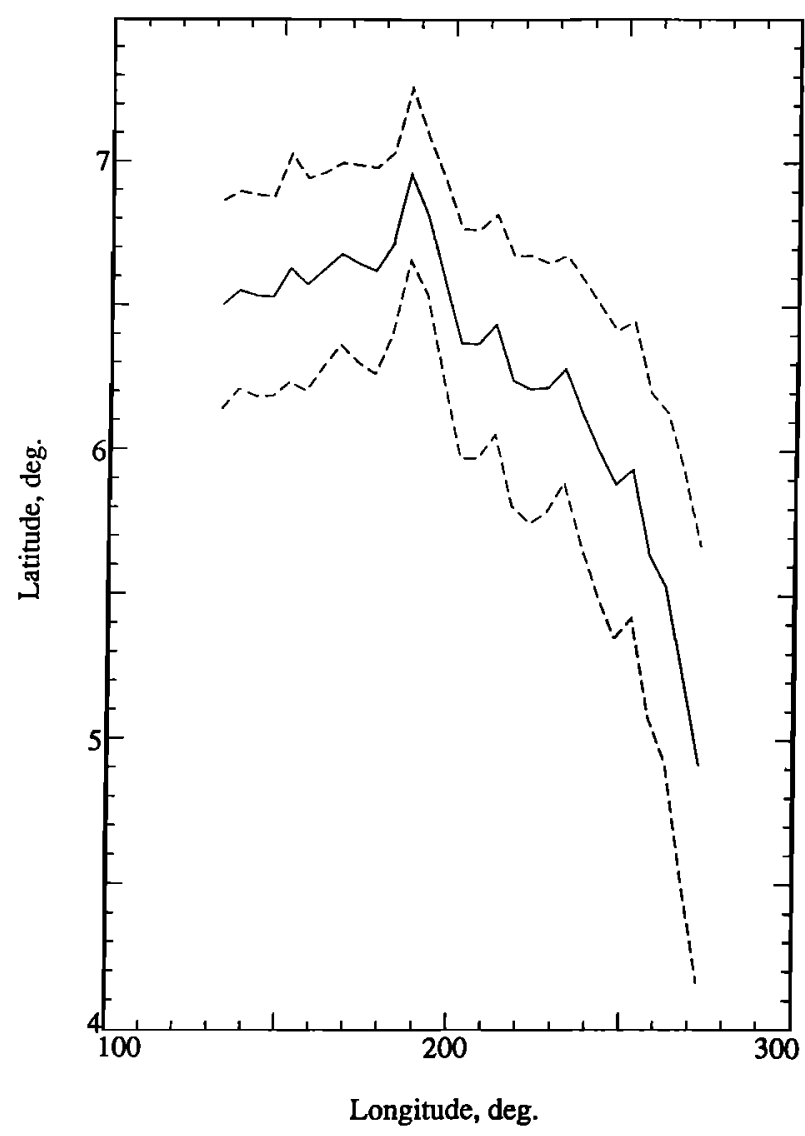

Figure 6. The same as Figure 4, except for S2. 


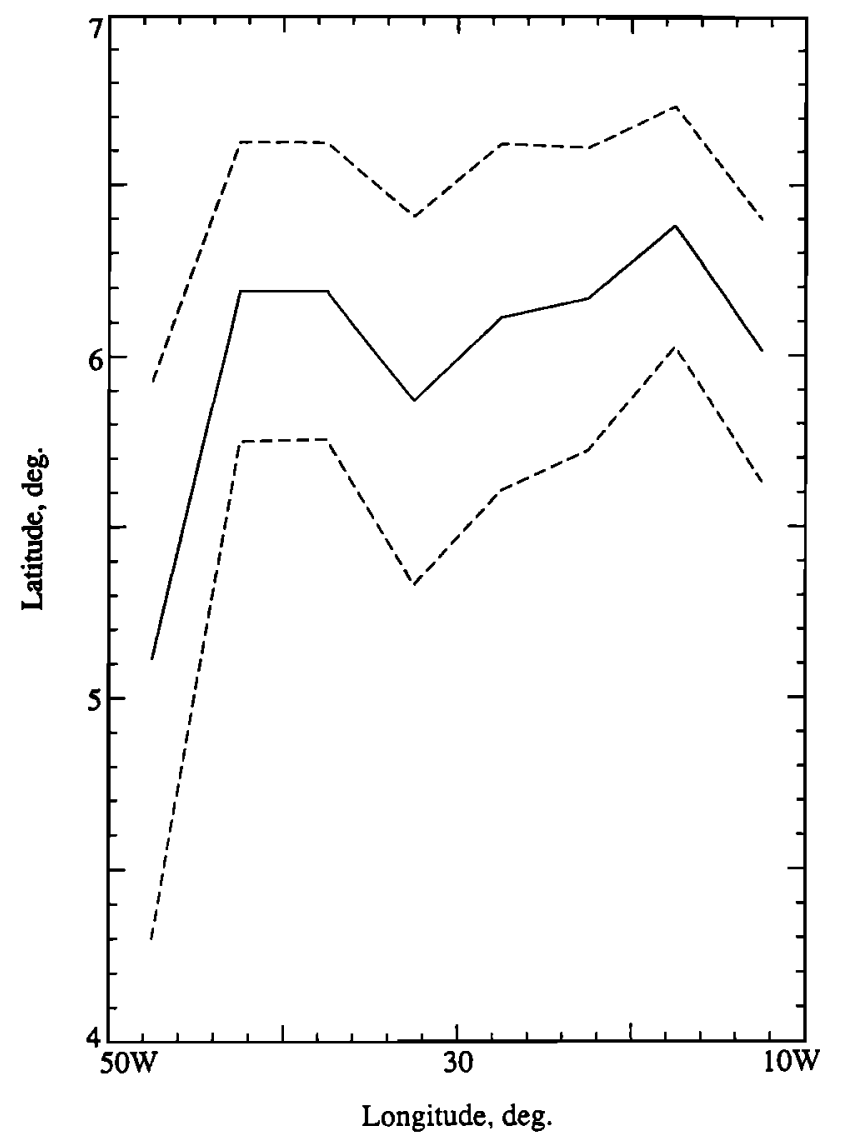

Figure 7. The same as Figure 5, except for S2.

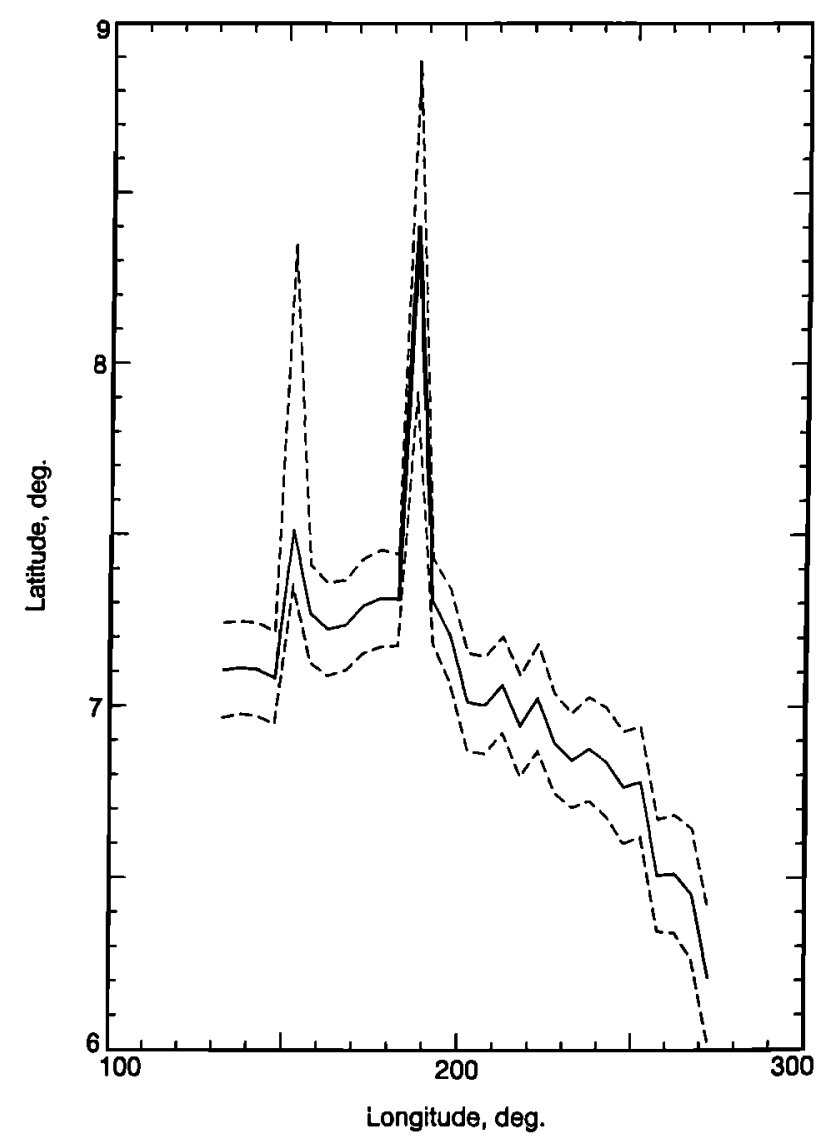

Figure 8. The same as Figure 6, except for T/P.

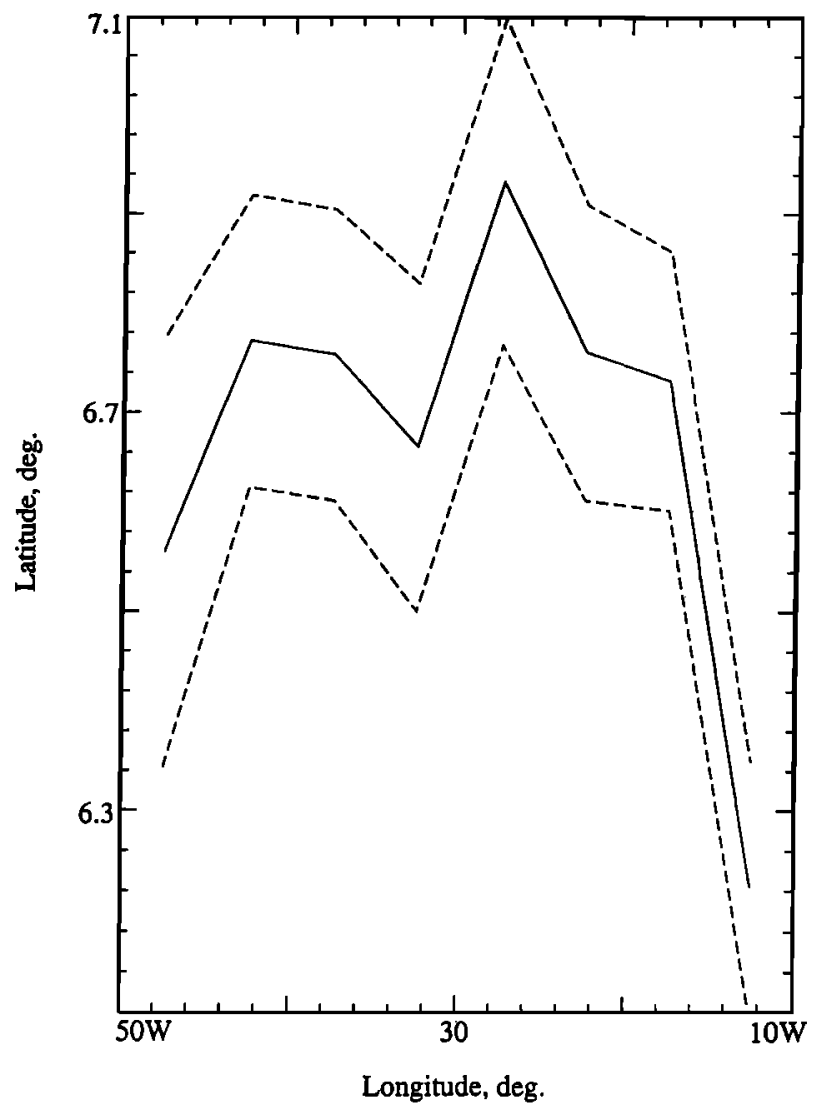

Figure 9. The same as Figure 7, except for T/P.

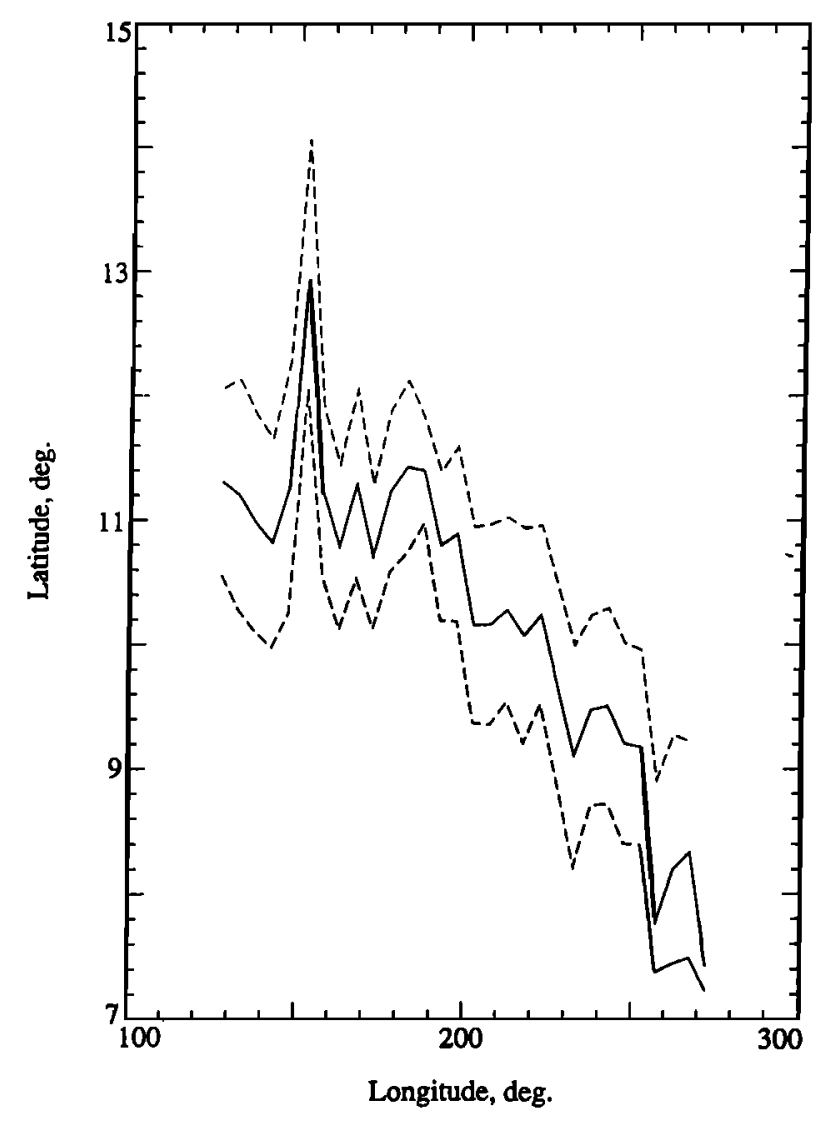

Figure 10. The same as Figure 4, except for ERS 


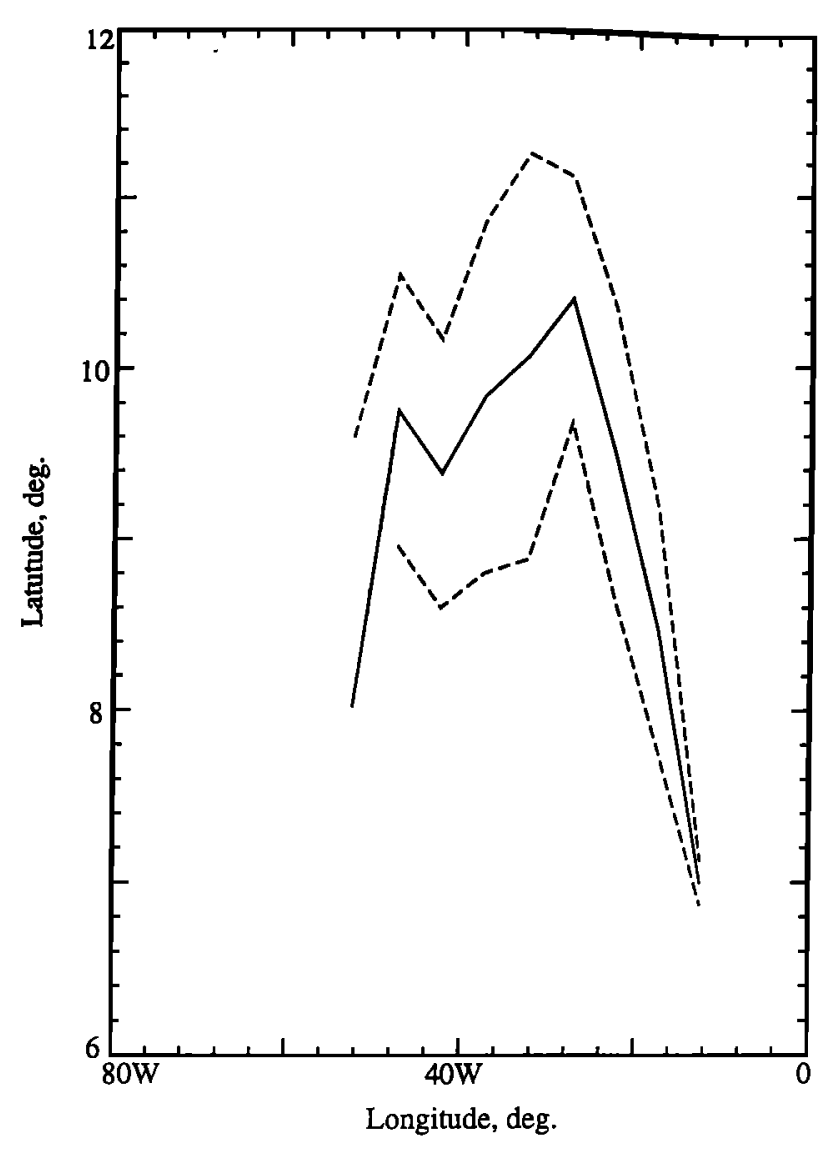

Figure 11. The same as Figure 5, except for ERS

The near-4 day internal gravity wave mode will alias to a near-21-day period for $T / P$ and near 68 days for Geosat. The alias period for $T / P$ is near the alias periods of several smaller tides including $\mathrm{L} 2$ and $\mu 2$. The alias period for Geosat is near the Mf tide.

Equatorial Kelvin waves are characterized by a nearly uniform eastward speed of propagation of about 2.8 $\mathrm{m} / \mathrm{sec}$ irrespective of frequency. Thus they may be considered more as traveling blobs than traditional waves. By looking at the speed of propagation of tidal aliases in Table 4 it is clear that for Geosat the K2 tidal alias will be indistinguishable from Kelvin waves.

Problems related to Kelvin waves can also arise for $T / P$. Plate 1 shows a time-longitude plot of the equatorial Pacific created from a $0.25^{\circ}$ gridded version of $\mathrm{T} / \mathrm{P}$ data. The gridded data was produced by using $\mathrm{a}$ two-level Cressman analysis with radii of influence of 600 and $100 \mathrm{~km}$, respectively [Hendricks et al., 1996]. Near Chile there is a westward propagating wave that is consistent with intraseasonal Kelvin waves reflecting from the coastline as westward propagating Rossby waves. The westward propagating wave largely terminates at the Galapagos Islands as would be dynamically expected. Oceanic intraseasonal variability peaks at periods near 60-75 days [Kessler et al., 1995]. The reflection coefficient for such waves is about 0.6 [Clarke, 1992], and so it is plausible that such reflections are taking place.
Plate 2 shows the region between the Galapagos and Chile with the mean subtracted at each successive time. This removes the annual signal and accentuates the westward propagating waves. Figure 12 shows a power spectrum from this region after removing long-period variations with wavelet analysis [Coffey et al., 1997]. The peak corresponding to the westward propagation is readily apparent. As might be expected from $\mathrm{T} / \mathrm{P}$ data, the frequency is better determined than the wavelength. The wavelength is consistent with westward propagating interseasonal Rossby waves.

The only clue that these may not be Rossby waves is that the pattern is a little too regular. In fact, they are dominantly due to a near-coastal error in the Desai and Wahr [1995] tidal model used to correct the data. What is curious is that the data reflects the secondary westward propagating alias and not the expected eastward propagation. This occurs because the analysis used preserves as much fine scale as possible near the spatial Nyquist. If energy near the Nyquist is smoothed as suggested by Schlax and Chelton [1994], this westward propagating alias would be largely filtered out. This example illustrates the importance of understanding all possible alias characteristics that may occur and the effect that analysis schemes will have.

\section{Sampling Nonoceanographic Phenomena}

Unmodeled errors in the altimeter measurement can lead to aliased signals if changes occur more rapidly than the Nyquist frequency. For each altimetric correction the altimeter range is estimated by some combination of measurement and modeling. Errors will appear as deviations of the sea surface.

Nonoceanographic phenomena that contribute to the altimeter measurement include the dry troposphere, wet troposphere, ionospere, tracker bias, electromagnetic bias, and orbit error. Here, as an example, we discuss aliasing caused by subsampling the dry troposphere.

Much of the temporal energy changes in the atmosphere occur at frequencies higher than the Nyquist frequency for altimetric measurements, and higher frequencies provide a significant contribution to the errors in the atmospheric corrections. Figure 13 shows the effect of sampling the European Centre for MediumRange Weather Forecasts (ECMWF) 6 hour mean pressures at the $T / P$ repeat period of near 10 days. The sampled signal has been offset vertically by 2 mbar for clarity. A 1 mbar pressure change corresponds to a static sea level change of $1 \mathrm{~cm}$ owing to the inverse barometer effect and a change in the radar path delay of $0.23 \mathrm{~cm}$. By comparing the original to the sampled signal, centimeter level aliasing can be observed.

\section{Conclusions}

There are many ways in which the behavior of sampled phenomena can lead to problems in the interpreta- 

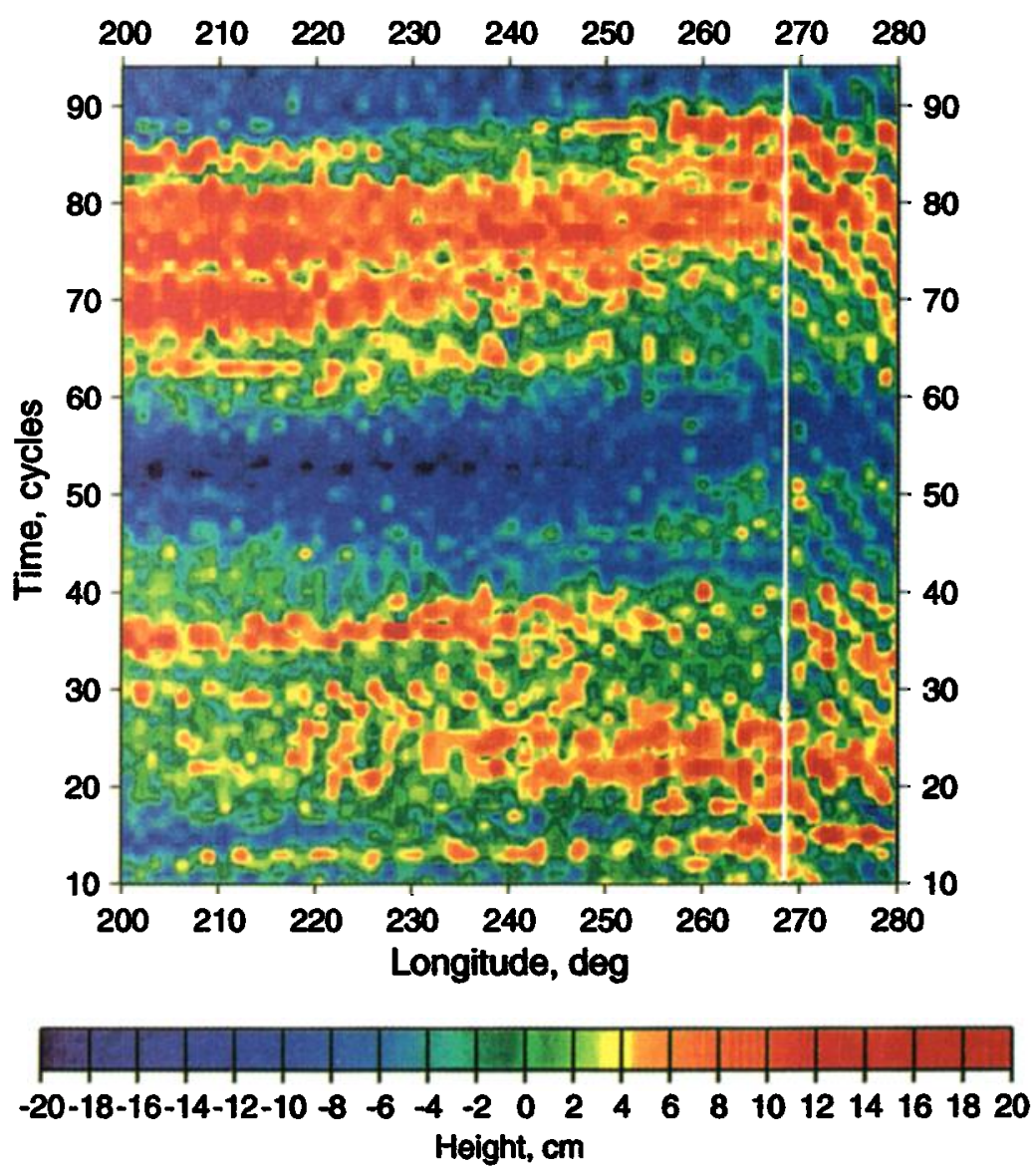

Plate 1. Time longitude plot of sea level along the equator. Note the westward propagating waves apparently reflecting from the Chile coast and ending near the Galapagos Islands.

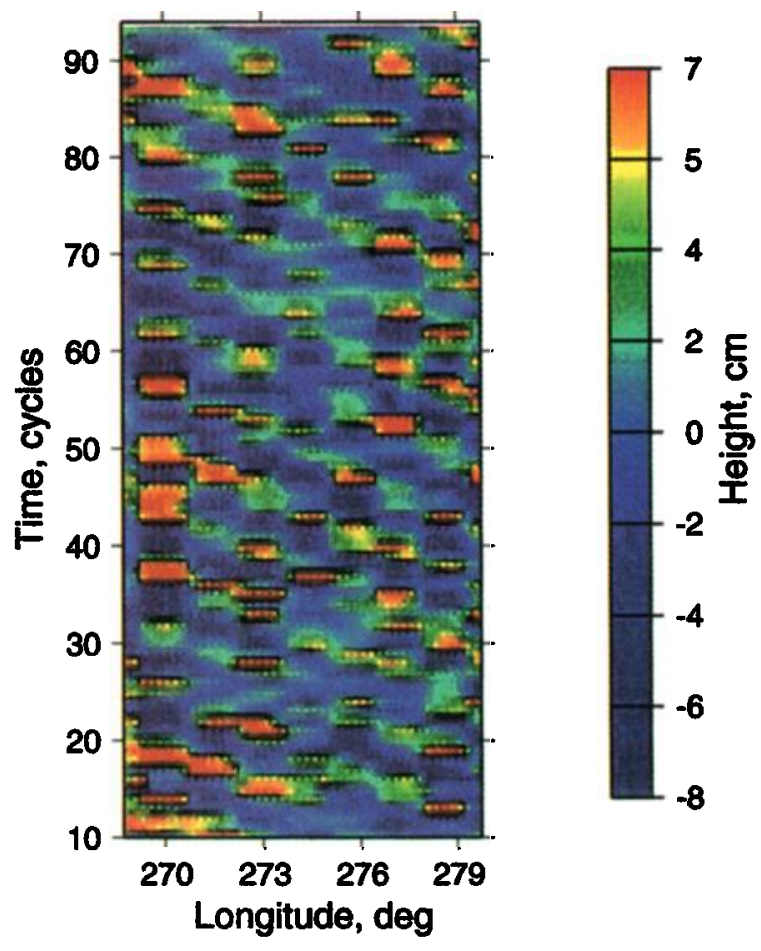

Plate 2. An expanded version of Figure 10 for the area between the Galapagos and the Chile coast with the mean signal removed at each time to accentuate the westward propagating waves.

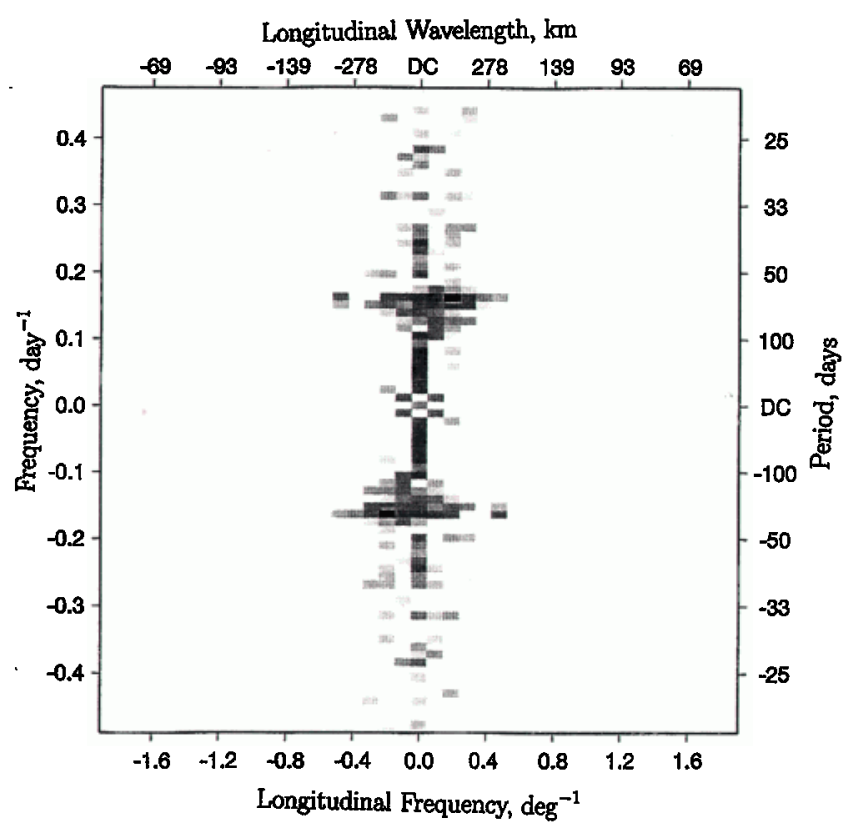

Figure 12. A power spectrum of the region shown in Figure 11 with long-term variations removed by wavelet analysis. 


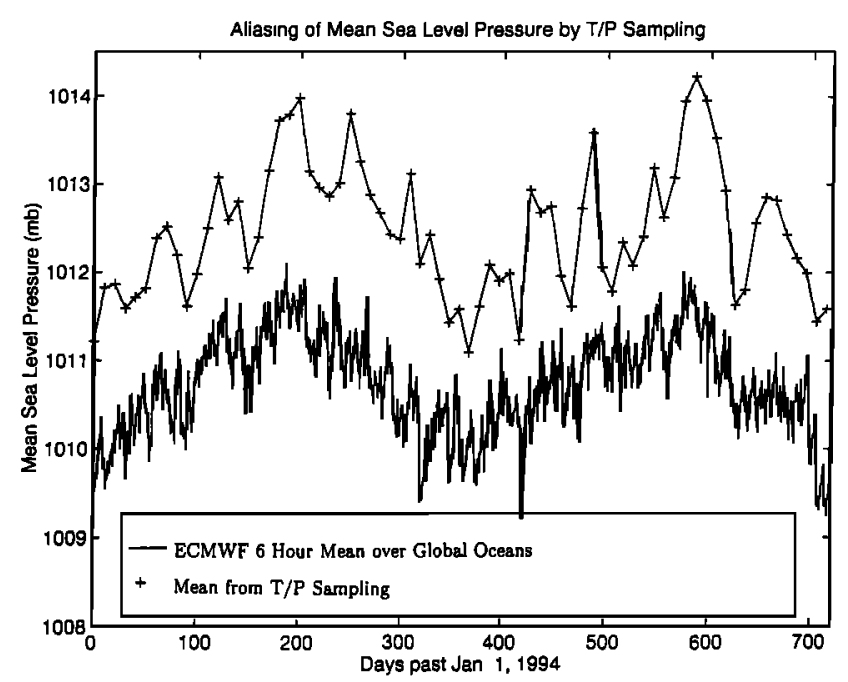

Figure 13. Aliasing of mean sea level presure by $T / P$ sampling. The $\mathrm{T} / \mathrm{P}$ sampled data has been offset vertically by 2 mbar for clarity

tion of altimetry data. Most aliasing problems can be resolved if the nature of the problem is understood. The examples given in this paper are intended to illustrate the types of problems that can arise.

The simplest problems that arise are those that occur because of misidentification of patterns. Because many phenomena appear in the altimeter data with both radically different frequency and wavenumber from the underlying phenomena, it is easy to misidentify them. For example, until recently, the trackiness that is often found with aliased tidal signals was sometimes mistaken for orbit error. Now that trackiness has been associated with tide errors, almost any trackiness is first attributed to tides whether it is or not.

Problems can also occur in situations where the underlying dynamics are not understood. One must be extremely careful in inferring the characteristics of the unknown phenomena. It is dangerous to subjectively watch movies of gridded altimeter data and infer the ocean response.

More complicated is the situation illustrated by the M2 tide and first mode baroclinic Rossby waves where the frequency and wavenumber of an aliased phenomena match the dispersion relation of some other oceanographic phenomena. In this case, partial separation of the signals can be obtained by noting that the aliased phenomena will have a different direction of propagation for ascending and descending tracks, while the unaliased phenomena will appear the same for ascending and descending tracks. Separation will be best at latitudes where the ascending and descending tracks are nearly perpendicular to one another and will be worst near the equator and near the turning latitudes where ascending and descending tracks are most nearly parallel. It is for this reason that it is important to not just consider the east-west component of propagation at midlatitudes, since this will be the same for both ascending and descending tracks.

The worst situation occurs when the frequency and wavenumber of two aliased phenomena are very close to each other. In this case, separation based on the data at such a location may not be possible. For this situation, more extreme measures need to be taken, such as making solutions for surrounding areas and extending them into the problem region via modeling or other techniques.

As has been shown in the preceding sections, the signals seen in altimeter data can be radically different from the underlying phenomena that were sampled. Because the nature of the real-world signals is dependent on the topography and stratification of the ocean, it is impossible to cover all examples of signals that will create problems in analysis and interpretation. This is especially true in coastal regions where frequencies and wavenumbers are generally higher.

Understanding the sampling characteristics of altimeter data can help attribute unknown signals to the correct source. In areas where known signals exist, possible sampling conflicts can be determined ahead of time. By developing proper analysis techniques, advantage can be taken of the fact that the spatial propagation of aliased signals will in general be different on ascending and descending ground tracks.

While it is not practical to achieve the ideal of sampling fine enough in space and time to avoid all aliasing problems, use of multiple satellites can help the situation. Currently, T/P, ERS-1, and ERS-2 are flying, and GFO will launch in late 1997. Proper combinations of data from these missions can help identify and eliminate aliasing problems associated with any individual data set. In fact, the design of future altimetric missions should be strongly influenced by the need to minimize problems associated with aliasing.

Acknowledgments. This work was performed under contract NA36GP0453 with the National Oceanic and Atmospheric Administration.

\section{References}

Bernstein, R.L., R.H. Whritner, and G. Born, Determination of ocean current variability using Seasat altimeter data, Eos Trans. $A G U, 60(18), 232,1979$.

Cartwright, D.E., and R.D. Ray, Oceanic tides from Geosat altimetry, J. Geophys. Res., 95(C3), 3069-3090, 1990.

Chase, R., and M. Mundt, On optimizing a constellation of altimetric satellites for measuring global oceanic mesoscale, J. Atmos. Sci., 37(4), 477-489, 1989.

Clarke, A.J., Low frequency reflection from a nonmeridional eastern ocean boundary and the use of coastal sea level to monitor eastern Pacific equatorial Kelvin waves, J. Phys. Oceanogr., 22, 163-183, 1992.

Coffey, M., Boundary-compensated wavelet bases, IEEE Trans. Acoust. Speech and Signal Process., 3, 2129-2132, 1997.

Desai, S.D., and J.M. Wahr, Empirical ocean tide models estimated from TOPEX/POSEIDON altimetry, J. Geophys. Res., 100(C12), 25205-25228, 1995. 
Emery, W.J., W.G. Lee, and L. Magaard, Geographic and seasonal distributions of Brunt-Vaisala frequency and Rossby radii in the North Pacific and North Atlantic, J. Phys. Oceanogr., 14, 294-317, 1984.

Groves, G.W., and M. Miyata, On weather-induced long waves in the equatorial Pacific, J. Mar. Res., 24, 115$128,1967$.

Hendricks, J.R., R.R. Leben, G.H. Born, and C.J. Koblinsky, EOF analysis of global TOPEX/POSEIDON altimeter data and implications for detection of global sea level rise, J. Geophys. Res., 101(C6), 14131-14145, 1996.

Jacobs, G.A., G.H. Born, M.E. Parke, and P.C. Allen, The global structure of the annual and serniannual sea surface height variability from Geosat altimeter data, J. Geophys. Res., $97(\mathrm{C11})$, 17813-17828, 1992.

Kessler, S. W., M.J. McPhaden, and K.M. Weickmann, Forcing of intraseasonal Kelvin waves in the eqatorial $\mathrm{Pa}$ cific, J. Geophys. Res., 100(C7), 10613-10631, 1995.

Matsumoto, K., M. Ooe, T. Sato, and J. Segawa, Ocean tide model obtained from TOPEX/POSEIDON altimetry data, J. Geophys. Res., 100(C12), 25319-25330, 1995.

Miyata, M., and G.W. Groves, Note on sea level observations at two nearby stations, J. Geophys. Res., 73(C12), 39653967, 1968.

Parke, M.E., R.H. Stewart, D.L. Farless, and D.E. Cartwright, On the choice of orbits for an altimetric satellite to study ocean circulation and tides, J. Geophys. Res., 92(C11), 11693-11707, 1987.

Parke, M.E., C. Tierney, and G. Born, Tide model evalu- ation: Final report, working paper for the TOPEX/POSEIDON Science Working Team tide model study, Rep. CCAR-9501, Cent. for Astrodyn. Res., Univ. of Colo., Boulder, 1995.

Philander, S.G., El Nino, La Nina, and the Southern Oscillation, 293 pp., Academic, San Diego, Calif., 1990.

Schlax, M.G., and D.B. Chelton, Aliased tidal errors in TOPEX/POSEIDON sea surface height data, J. Geophys. Res., , 99(C12), 24761-24776, 1994.

Shum, C.K., et al., Accuracy assessment of recent ocean tide models, J. Geophys. Res., 102, 25173-15194, 1997.

Tierney, C.C., M.E. Parke, and G.H. Born, Ocean tides from along-track altimetry, $J$. Geophys. Res., in press, 1998.

Wunsch, C., and A.E. Gill, Observations of equatorially trapped waves in Pacific sea level variations, Deep Sea Res. Oceanogr. Abstr., 23, 371-390, 1976.

G. H. Born, B. Leben, C. McLaughlin, and C. Tierney Colorado Center for Astrodynamics Research, Campus Box 431, University of Colorado, Boulder, CO 80309. (e-mail: georgeb@orbit.colorado.edu)

M.E. Parke, Department of Civil and Environmental Engineering and Geodetic Science, The Ohio State University, 2070 Neil Ave., Columbus, OH 43210. (e-mail: mparke@geodesy.eng.ohio-state.edu)

(Received July 8, 1996; revised July 21, 1997; accepted July 30, 1997.) 